\title{
Competencias relevantes en contaduría pública y finanzas: ¿existe consenso entre empleadores, profesores y estudiantes?
}

\author{
Relevant competences in public accounting and finance: Is there a \\ consensus among employers, professors and students?
}

Javier Montoya del Corte ${ }^{\mathrm{a}^{*}}$ y Gabriela María Farías Martínez

${ }^{a}$ Universidad de Cantabria, España

${ }^{b}$ Tecnológico de Monterrey, México

Recibido el 11 de noviembre de 2016; aceptado el 28 de marzo de 2017

Disponible en Internet el 30 de mayo de 2018

\section{Resumen}

El artículo busca analizar qué competencias son relevantes para el adecuado desempeño profesional de la Contaduría Pública y las Finanzas (CPF) desde la perspectiva del mercado -empleadores- y de la universidad -profesores y estudiantes-, y comprobar en qué medida existe coincidencia. Para lograr este fin, se llevó a cabo una investigación cuantitativa basada en una encuesta online. Posteriormente, se realizó una investigación cualitativa basada en un grupo focal. La información recabada se analizó con apoyo en los programas SPSS y NVivo, respectivamente. La evidencia obtenida muestra la existencia de un consenso general acerca de la mayor trascendencia de los valores, la ética y las actitudes, por encima de las habilidades y, sobre todo, de los conocimientos. No obstante, se identifican algunas diferencias significativas que revelan ciertas desviaciones entre la formación universitaria y los requerimientos del mercado laboral en la actualidad. Es por esto que se advierte la necesidad de alinear en mayor medida la formación académica con las demandas y requisitos del mercado laboral aumentando el acercamiento entre la universidad y el ámbito empresarial, así como poner un mayor énfasis en el aprendizaje autónomo de los estudiantes.

Códigos JEL: A22, G00, M40.

Palabras clave: Formación de competencias; Universidad; Empleabilidad; Contaduría pública; Finanzas.

*Autor para correspondencia

Correo electrónico: montoyaj@unican.es (J. Montoya del Corte)

La revisión por pares es responsabilidad de la Universidad Nacional Autónoma de México. 


\begin{abstract}
This article intends to analyze what competences are relevant for the adequate professional performance of the Public Accounting and Finances (CPA) from the perspective of the market -employers- and the university -professors and students-, and verify to what extent there is agreement. To achieve this objective, a quantitative investigation based on an online survey was made. Afterwards, a qualitative research based on a focus group was conducted. The collected information was analyzed with the support of the SPSS and NVivo program, respectively. The obtained evidence shows the existence of a general consensus on the major importance of values, ethics and attitudes, above skills and, primarily, above knowledge. However, some significant differences that reveal certain deviations between university education and labor market requirements were identified. That is why this article advises of the need to align in greater measure the academic education with the demands and requirements of the working market by increasing the approach between universities and business fields, as well as putting major effort in the students' self-sufficient learning processes.
\end{abstract}

JEL Classification: A22, G00, M40.

Key words: Skills training; College; employability; Public accounting; Finance.

\title{
Introducción
}

Durante las últimas décadas, la globalización y el desarrollo tecnológico destacan como factores determinantes de cambios cada vez más rápidos y trascendentes. Como avanzaba Delors (1996), para lograr una adecuada adaptación a dichos cambios, el mercado laboral exige profesionales altamente cualificados en la conjunción de tres vertientes complementarias: la cognitiva ("saber"), la procedimental ("saber hacer") y la actitudinal ("saber ser y estar"). Ante esta situación, el sistema universitario se ha visto obligado a reaccionar, llevando a cabo una reforma estructural que permita aproximar la oferta académica a las demandas de la sociedad actual, incrementando así la empleabilidad de sus egresados. En este punto, es donde surge el paradigma de la formación basada en competencias (Martínez y Echeverría, 2009), término que abarca el conjunto de conocimientos, habilidades y valores, ética y actitudes que determinan la capacidad de una persona para responder adecuadamente a las exigencias de un puesto de trabajo concreto (Biemans, Nieuwenbuis, Poell, Mulder y Wesselink, 2004).

El objetivo de la presente investigación es profundizar en el análisis de la formación de competencias relevantes en el ámbito específico de la Contaduría Pública y las Finanzas (CPF) para el caso particular de México, donde existe un vacío en la literatura empírica previa (Montoya, Farías y Mancina, 2013). Para ello, se procede de la siguiente manera. En primer lugar, se selecciona un conjunto de conocimientos, habilidades y valores, ética y actitudes que la normativa internacional y la literatura previa han destacado como importantes para el adecuado desempeño profesional en estas disciplinas. A continuación, se comparan los puntos de vista del mercado, representado por los profesionales ejercientes (empleadores), y de la universidad, representada por los académicos que desempeñan su labor en las Instituciones de Educación Superior (profesores) y por las personas que están cursando una Licenciatura a nivel de Grado (estudiantes). El propósito de realizar esta diferenciación es determinar la existencia de un posible gap en las percepciones de los tres colectivos con respecto a las tres vertientes que integran el término competencia. 
El estudio empírico que se realiza está basado en una metodología de investigación de naturaleza mixta, que combina una primera etapa cuantitativa con otra posterior cualitativa. En primer lugar, se realiza una encuesta por Internet a los profesionales miembros del Instituto Mexicano de Contadores Públicos (IMCP) y del Instituto Mexicano de Ejecutivos de Finanzas (IMEF), así como a profesores y estudiantes de la Licenciatura en Contaduría Pública y Finanzas (LCPF) de diversas universidades mexicanas. A continuación, con el ánimo de poder enriquecer y agregar valor a la interpretación de la información recopilada, se lleva a cabo un grupo focal en el que participan representantes de los tres colectivos encuestados.

Entre los principales resultados obtenidos cabe resaltar la existencia de un consenso general acerca de la mayor trascendencia que los tres grupos de interés estudiados otorgan a los valores, la ética y las actitudes, por encima de las habilidades y, sobre todo, de los conocimientos. No obstante, se identifican algunas diferencias significativas, fundamentalmente inter-grupos, que revelan ciertas desviaciones entre el nivel de formación ofrecido por la universidad y el requerido por el mercado laboral en la actualidad. Con este estudio se contribuye a la escasa literatura empírica existente sobre este tema en Latinoamérica, en general, y en México, más en particular, y tiene importantes implicaciones prácticas tanto en el ámbito académico como en el gerencial.

Lo que resta de trabajo se estructura de la siguiente manera. Tras esta introducción, se revisa brevemente los fundamentos teóricos que, tanto a nivel normativo como empírico, sirven para contextualizar el tema estudiado. A continuación, se explican más ampliamente los aspectos metodológicos de la investigación realizada. En el cuarto apartado, se analizan los resultados obtenidos. Finalmente, se presentan las principales conclusiones alcanzadas, junto con las limitaciones y futuras líneas de investigación que se derivan, y se referencia la bibliografía consultada.

\section{Marco normativo y literatura previa sobre competencias en CPF}

Con el propósito de lograr el éxito profesional de los egresados universitarios en un mercado laboral cada vez más global, cambiante y dinámico, ya desde finales del pasado siglo algunos expertos comenzaron a promover la trascendencia del enfoque de la formación basada en competencias en general (McLagan, 1997; Bennett, Dunne y Carré, 1999), y en la práctica gerencial de la administración de empresas más en particular (Varela, 2003). Dentro de la CPF, disciplinas que ocupan la atención de este trabajo, se han erigido en un importante referente a nivel mundial las denominadas International Education Standards (IES), emitidas por el International Accounting Education Standards Board (IAESB) de la International Federation of Accountants (IFAC).

Este marco normativo define el término competencia como "la capacidad de una persona para ejecutar un trabajo cumpliendo con un determinado estándar en entornos laborales reales". Asimismo, define el término capacidad como "el conjunto formado por los conocimientos, habilidades, y valores, ética y actitudes profesionales requeridos para demostrar competencia" (IFAC, 2014, pp. 20-21). De estas dos definiciones se desprende, por tanto, que para ser competentes los profesionales deben estar capacitados en tres vertientes diferentes y complementarias: la cognitiva (conocimientos -técnicos-), la procedimental (habilidades -destrezas-) y la actitudinal (valores, ética y actitudes -comportamiento-).

A este respecto, la IFAC (2014) abunda ofreciendo una serie de orientaciones sobre los 
aspectos específicos que deben atenderse en cada una de estas tres vertientes. Esto lo hace en los documentos que se señalan a continuación, que junto con la literatura previa citada más adelante fueron tomados como referencia al momento de elaborar el instrumento de recogida de información para la investigación cuantitativa de este trabajo:

- IES 2. Initial professional development. Technical competence.

- IES 3. Initial professional development. Professional skills.

- IES 4. Initial professional development. Professional values, ethics, and attitudes.

Con relación a la producción académica sobre esta línea de investigación, dentro del presente siglo han proliferado los trabajos orientados a determinar cuáles deben ser los objetivos y las funciones de la universidad como Institución de Educación Superior, de tal manera que se esclarezca lo que la sociedad le puede, y le debe, demandar (Rubio, 2005). A este respecto, existe un consenso general acerca de que una de las tareas principales que le corresponde realizar a la universidad es contribuir a la empleabilidad de sus egresados, lo que implica, precisamente, desarrollar y fortalecer las competencias que en cada disciplina se consideren necesarias (Caballero, López, y Lampón, 2014). Centrando la atención en el ámbito general de la administración de empresas, y de forma específica en el de la CPF, existe una amplia y diversa evidencia empírica a nivel internacional que sustenta que los empleadores que operan en el mercado laboral consideran que los egresados universitarios no están suficientemente preparados en las distintas vertientes de las competencias requeridas.

Dentro del mundo anglosajón, en Australia Kavanagh y Drennan (2008) ponen de manifiesto que los empleadores echan en falta, sobre todo, un mejor conocimiento del mundo real y de los negocios, así como un buen entendimiento de los conceptos básicos contables y mayores habilidades analíticas; Pan y Perera (2012) evidencian la necesidad de fomentar una mejor comprensión de cómo la contabilidad debe ser interpretada y cómo puede ser utilizada para mejorar un negocio, así como una mayor capacidad para resolver problemas y gestionar el tiempo; y Tempone, Kavanagh, Segal, Hancock, Howieson y Kent (2012) evidencian que los empleadores valoran especialmente la habilidad de los egresados universitarios para comunicarse, trabajar en equipo y auto-gestionarse. En Estados Unidos, igualmente, Lin, Krishnan y Grace (2013) obtienen que los empleadores demandan a los egresados universitarios una mayor capacidad para comunicarse verbalmente y por escrito, así como mayores habilidades interpersonales para relacionarse con los demás. Por último, en el Reino Unido, Senik, Broad, Mat y Kadir (2013) revelan una falta de formación en la comprensión básica y el manejo de software específico sobre contabilidad, auditoría e impuestos.

Dentro del contexto iberoamericano, en España Arquero (2000) y Arquero, Donoso, Jiménez y González (2009) evidencian importantes deficiencias formativas en varias habilidades notécnicas, especialmente la comunicación oral y escrita y la resolución de problemas; Marzo, Pedraja y Rivera (2009) ponen de manifiesto que la formación práctica recibida por los egresados universitarios es donde mayor desviación existe. Además, estos tienen importantes carencias en habilidades tales como iniciativa y comunicación oral (sociales), aplicación práctica del conocimiento, creatividad, adaptación a nuevas situaciones y aplicación de normas y regulaciones (metodológicas), establecimiento de relaciones con cargos superiores, negociación, resolución de conflictos, actitud resolutiva y corporativa ante situaciones complicadas y capacidad para motivar a los demás (participativas); Pujol-Jover, Riera-Prunera y Abio (2015) obtienen que 
los empleadores encuentran algunas carencias en la capacidad de los egresados universitarios para gestionar adecuadamente el tiempo, adaptarse a los cambios, comunicarse, trabajar en equipo y tener visión empresarial; y Bartual y Turmo (2016) ponen de relieve la importancia otorgada por los empleadores a de las competencias genéricas. No obstante, se observan diferencias valorativas según la tipología de empresas. Asimismo, se evidencian déficits en algunos aspectos relevantes, como la formación práctica y la capacidad de iniciativa, de análisis o de organización. En Colombia, Cabeza, Castrillón y Lombana (2012) encuentran que se deben desarrollar las competencias relacionadas con las tecnologías de la información y la comunicación, la capacidad crítica y autocrítica, la capacidad de abstracción, análisis y síntesis, la gestión del riesgo y del tiempo, la confrontación de nuevas situaciones, el compromiso con el ambiente y la apertura hacia contextos internacionales; por su parte, Castrillón, Cabeza y Lombana (2015) identifican las cinco competencias genéricas y específicas más importantes para los empleadores en la disciplina administrativa. Como genéricas señalan: compromiso ético, compromiso con la calidad, capacidad de tomar decisiones, capacidad para identificar, formular y resolver problemas y trabajo en equipo. Como específicas indican: desarrollar la planificación táctica, operativa y estratégica, utilizar el liderazgo para el logro de la meta de la organización, gestionar y desarrollar el talento humano en la organización, identificar y optimizar los procesos de negocios de las organizaciones e interpretar la información contable y financiera para la toma de decisiones gerenciales. Además, obtiene que los empleadores consideran que se deben fortalecer especialmente la identificación y optimización de los procesos de negocio de las organizaciones y la detección de oportunidades para emprender nuevos negocios o desarrollar nuevos productos.

\section{Metodología}

Para la obtención de la información necesaria se consideró conveniente el uso de una metodología de investigación de naturaleza mixta, por lo que se combinó la realización de una encuesta a través de un cuestionario estructurado y auto-administrado (cuantitativa) con un grupo focal (cualitativa).

\section{Diseño de la investigación cuantitativa: el cuestionario}

Tras revisar la normativa internacional y la literatura previa sobre el tema, se elaboró un instrumento que fue sometido a una prueba piloto en la que participaron 10 empleadores, 10 profesores y 20 estudiantes. La versión final del cuestionario estuvo integrada por cuatro bloques de preguntas referentes a las características socio-demográficas de los sujetos y al conjunto de conocimientos, habilidades y valores, ética y actitudes (competencias) que, a priori, se consideran relevantes para el ejercicio profesional de la CPF. Aunque se utilizó el mismo instrumento, se elaboraron tres versiones para adaptarlo a cada uno de los colectivos participantes: a los empleadores se les preguntó por la competencias requeridas para el ejercicio de la CPF, a los profesores por las competencias en las que se centraba la formación de los estudiantes de CPF de sus universidades, y a los estudiantes por las competencias que habían logrado desarrollar durante sus estudios de CPF. La valoración de las competencias se capturó a través de una escala Likert de " $l=$ Poco" a " $5=$ Mucho". El cuestionario se aplicó de forma auto-administrada a través de Internet en mayo de 2015, con el apoyo de la Red Nacional 
de Educación e Investigación de la Corporación Universitaria para el Desarrollo de Internet (CUDI) en México.

Como ya se ha mencionado, la encuesta se dirigió a tres colectivos diferentes que se consideran primordiales para poder abordar adecuadamente el tema objeto de estudio. Como representantes del mercado, se toman los profesionales ejercientes que son socios del Instituto Mexicano de Contadores Públicos (IMCP) y del Instituto Mexicano de Ejecutivos de Finanzas (IMEF). Como representantes de la universidad, se toman los profesores de planta y los estudiantes de último curso de la LCPF de un total de 12 instituciones públicas y privadas del país. Se enviaron dos correos electrónicos a los integrantes de estos colectivos, uno inicial y otro recordatorio a los quince días. En ellos se daba una breve explicación de la investigación y se facilitaba el enlace a la página Web donde estaba ubicado el cuestionario, a la que sólo se podía acceder con un nombre de usuario y una clave. Las respuestas de los participantes se iban registrando de forma automática en una base de datos. Tras depurarla, el número total de respuestas válidas recibidas para el mercado fueron 992, repartidas entre 899 socios del IMCP y 93 socios del IMEF; y para la universidad fueron 827, repartidas entre 134 profesores y 693 estudiantes.

Los resultados de fiabilidad o consistencia interna de las tres vertientes de las competencias fueron medidos a posteriori a través del Alfa de Cronbach, obteniendo los resultados que se muestran en la Tabla 1. De acuerdo con Nunnally (1978) y Segars (1997), se cuenta con niveles de confiabilidad elevados en el instrumento, puesto que el índice Alpha es mayor que 0.70 en todos los casos.

Tabla 1

Fiabilidad del cuestionario

\begin{tabular}{llllll}
\hline COMPETENCIAS & IMCP & IMEF & Profesores & Estudiantes & Total \\
\hline Conocimientos & 0.945 & 0.945 & 0.966 & 0.954 & 0.955 \\
\hline Habilidades & 0.964 & 0.960 & 0.981 & 0.971 & 0.973 \\
\hline Valores, ética y actitudes & 0.910 & 0.885 & 0.945 & 0.935 & 0.936 \\
\hline
\end{tabular}

Fuente: elaboración propia.

El análisis de resultados está basado en el estudio de las puntuaciones medias obtenidas para cada variable o indicador de los 24 conocimientos, 30 habilidades y 7 valores, ética y actitudes consideradas. Asimismo, se examinan las posibles diferencias entre colectivos mediante un análisis de la varianza (ANOVA), utilizando como factor de segmentación las variables mercado/universidad (IMCP+IMEF versus profesores+estudiantes) y tipo de sujeto (IMCP, IMEF, profesor y estudiante). Para llevar a cabo este análisis se utilizó el programa Statistical Package for the Social Sciences (SPSS) en su versión 22.

\section{Diseño de la investigación cualitativa: el grupo focal}

Con el ánimo de poder profundizar en la interpretación de los resultados obtenidos en la encuesta, en agosto de 2015 se organizó una sesión para el debate con un grupo focal siguiendo las etapas definidas por Ivankovich y Araya (2011). Para esta investigación se contó con la colaboración y los contactos de una universidad mexicana que previamente había participado 
en la encuesta, logrando conformar un grupo integrado por 10 personas, repartidos de la siguiente manera:

- Tres empleadores. Un Contador Público y Auditor ejerciente como Socio en una de las Big-4; un Contador Público, Auditor y Fiscalista ejerciente como Socio en un despacho de consultoría y asesoría fiscal de pequeña dimensión; y una Contadora Pública, Auditora y Fiscalista ejerciente como Directora de Administración en un Banco de gran dimensión.

- Tres profesores. Un profesor y Director de carrera de la LCPF; un profesor y Director de carrera de la Licenciatura en Administración Financiera; una profesora de la LCPF, que además dirige un pequeño despacho de consultoría administrativa.

- Cuatro estudiantes. Dos de la LCPF y otros dos de la Licenciatura en Administración Financiera.

La sesión fue moderada por los investigadores, planteándose dos cuestiones fundamentales para el debate sobre el tema objeto de estudio en este trabajo: $\left(1^{\circ}\right)$ nivel de relevancia de las tres vertientes de las competencias -conocimientos, habilidades y valores, ética y actitudespara el ejercicio de la CPF a nivel profesional; y $\left(2^{\circ}\right)$ formación que debería darse y se da a los estudiantes de CPF en la universidad. La duración de la sesión fue de una hora y quince minutos y su transcripción depurada ocupó un total de 10.032 palabras.

Para un análisis e interpretación de los resultados más exhaustivo se utilizó el programa informático de apoyo a la investigación cualitativa NVIVO en su versión 11. Éste ayuda a compilar, organizar y analizar contenido de entrevistas, discusiones de grupos focales, encuestas, audio, medios sociales y páginas web, pues cuenta con poderosas herramientas de búsqueda, consulta y visualización. Una vez introducida y codificada la información, se pueden identificar patrones, descubrir temas emergentes y hacer consultas de frecuencia de palabras, por ejemplo.

\section{Análisis de resultados}

\section{Análisis cuantitativo}

En la Tabla 2 se recogen las puntuaciones medias obtenidas para el conjunto de conocimientos, habilidades y valores, ética y actitudes considerados, organizando por columnas los resultados para el total de sujetos encuestados, para el mercado (empleadores del IMCP y del IMEF), para la universidad (profesores y estudiantes) y para cada uno de los colectivos individuales.

Tabla 2

Puntuaciones medias de las competencias

\begin{tabular}{llllllll}
\hline COMPETENCIAS & Total & Mercado & Universidad & IMCP & IMEF & Profesores & Estudiantes \\
\hline Conocimientos & 3.80 & 4.03 & 3.59 & 4.04 & 3.94 & 3.65 & 3.58 \\
\hline Habilidades & 4.06 & 4.35 & 3.82 & 4.35 & 4.32 & 3.68 & 3.85 \\
\hline Valores, ética y actitudes & 4.22 & 4.53 & 3.99 & 4.54 & 4.42 & 3.83 & 4.02 \\
\hline
\end{tabular}

Fuente: elaboración propia. 
Como puede observarse, en todos los casos resultan promedios más elevados para los valores, ética y actitudes, seguidos de las habilidades y a continuación de los conocimientos. Además, los promedios del mercado son superiores a los de la universidad en los tres casos, con niveles muy parecidos entre los dos colectivos que los integran. A efectos de profundizar en los análisis, en los próximos apartados se examinan por separado cada una de las tres vertientes de las competencias.

\section{Conocimientos}

En la Tabla 3 se presentan los resultados obtenidos con relación al nivel de conocimientos que un recién egresado universitario en CPF debe tener sobre un total de 24 materias para poder desempeñar adecuadamente su actividad profesional. Los datos están divididos según procedan de la opinión del mercado (empleadores del IMCP y del IMEF) o de la universidad (profesores y estudiantes), y ordenados de mayor a menor diferencia de medias entre ambos colectivos.

Se advierte que únicamente para "Mercadotecnia" el nivel de conocimientos es similar entre el mercado y la universidad, siendo la material menos importante y la cuarta menos relevante, respectivamente, en la formación de CPF. Así pues, 23 de las 24 materias consideradas en esta investigación presentan diferencias significativas entre el nivel de exigencia del mercado y el nivel de capacitación de la universidad, con puntuaciones medias superiores en todos los casos a favor del mercado. Este resultado muestra, por tanto, algunas debilidades de formación en conocimientos en CPF de las universidades en México actualmente, en particular en materias como "Contabilidad gubernamental", "Control de los sistemas de información basados en tecnología", "Riesgos asociados a los sistemas de información basados en tecnología" e "Impuestos", "Valores y ética profesionales", "Auditoría", "Contabilidad financiera y elaboración de reportes financieros" y "Finanzas y administración financiera".

No obstante, es necesario indicar que al estructurar las valoraciones medias de las materias por parte del mercado y de la universidad, las clasificaciones resultantes son muy parecidas en todos los casos. Las únicas materias en las que se identifica un mayor distanciamiento son "Control de los sistemas de información basados en tecnología" y "Riesgos asociados a los sistemas de información basados en tecnología", que se sitúan un poco más alto en el ranking del mercado que en el de la universidad. 


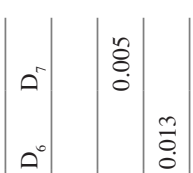

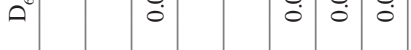
ค

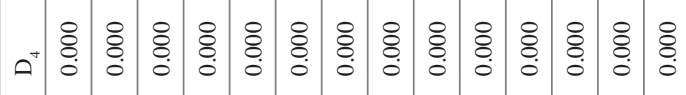

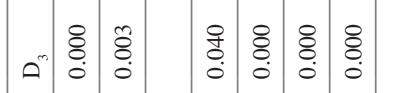
응 응

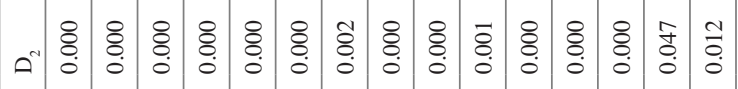
$-$

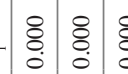
离

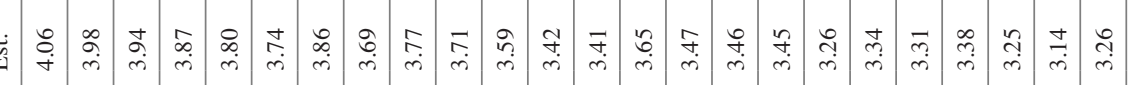

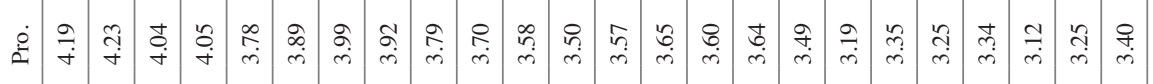

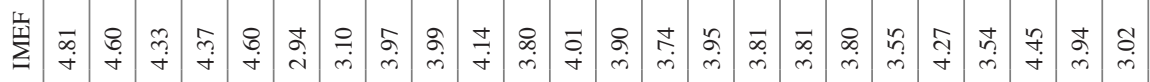

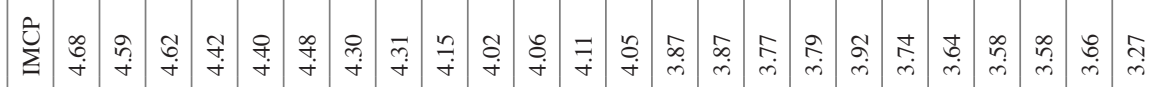

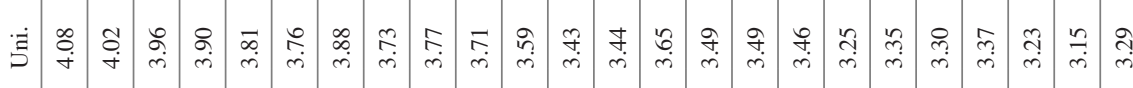

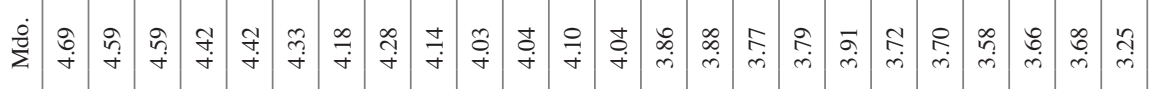
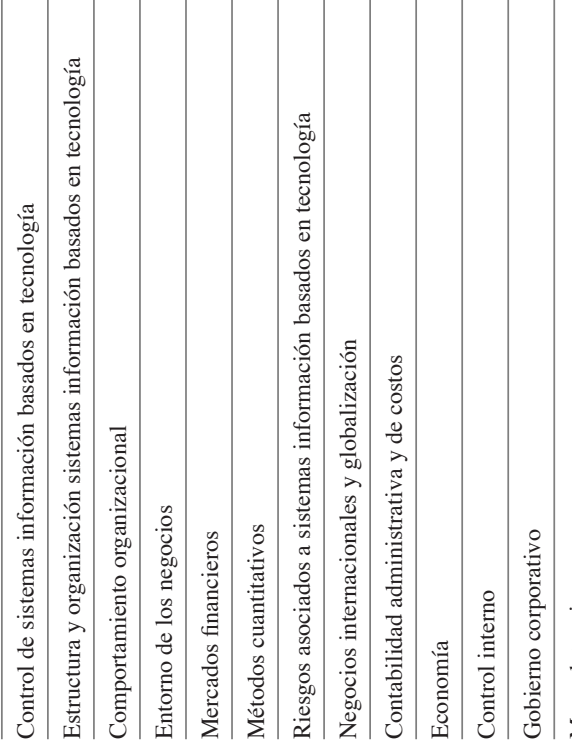

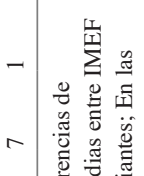

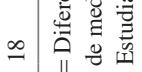

0 .

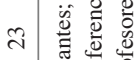

悉言

○

画

ส

o

중

त)

ㄴ.

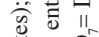

恶密

勇要通

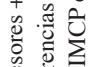

월

है동

粷命

刍

II. 을

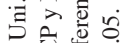

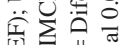

폴

$+\overline{0}$ is

ชิ 造

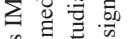

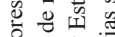

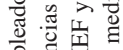

을

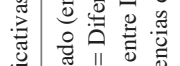

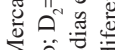

क

웡

$\sum \sum \mathrm{g}$

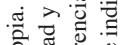

한

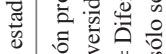

年 


\section{Habilidades}

En la Tabla 4 se presentan los resultados obtenidos con relación al nivel de destreza que un recién egresado universitario en CPF debe tener sobre un total de 30 habilidades para poder desempeñar adecuadamente su actividad profesional. Los datos están divididos según procedan de la opinión del mercado (empleadores del IMCP y del IMEF) o de la universidad (profesores y estudiantes), y ordenados de mayor a menor diferencia de medias entre ambos colectivos.

Se pone de manifiesto que las 30 habilidades consideradas en esta investigación presentan diferencias significativas entre el nivel de exigencia del mercado y el nivel de capacitación de la universidad. Excepto en el caso del "Uso de la tecnología para comunicarse", las valoraciones medias son superiores en el ámbito del mercado. De nuevo, este resultado muestra ciertas debilidades de formación en habilidades en CPF de las universidades en México actualmente, en especial en aspectos como el "Uso de la tecnología para resolver problemas", "Dominio del español y del inglés", "Dominio del español, del inglés y de algún otro idioma", "Comunicación escrita", "Administración del tiempo", "Aprendizaje continuo a lo largo de la vida" y "Razonamiento lógico".

Sin embargo, es necesario indicar que al estructurar las valoraciones medias de las habilidades por parte del mercado y de la universidad, las clasificaciones resultantes son muy parecidas en todos los casos. Las únicas habilidades en las que se identifica un mayor alejamiento son el "Uso de la tecnología para comunicarse", "Administrarse uno mismo", "Comunicación escrita" y "Comunicación oral". 


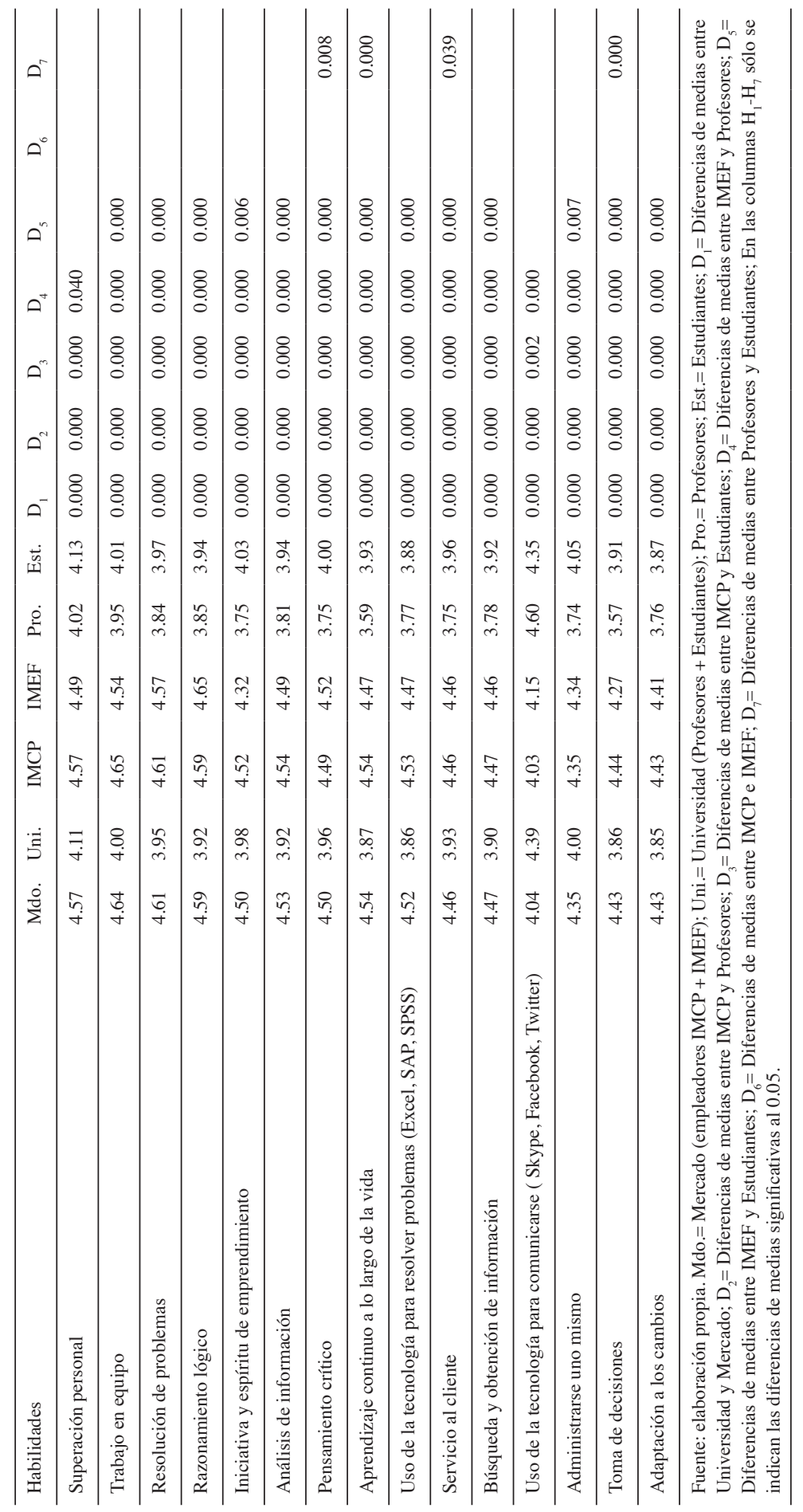



http://dx.doi.org/10.22201/fca.24488410e.2018.1265

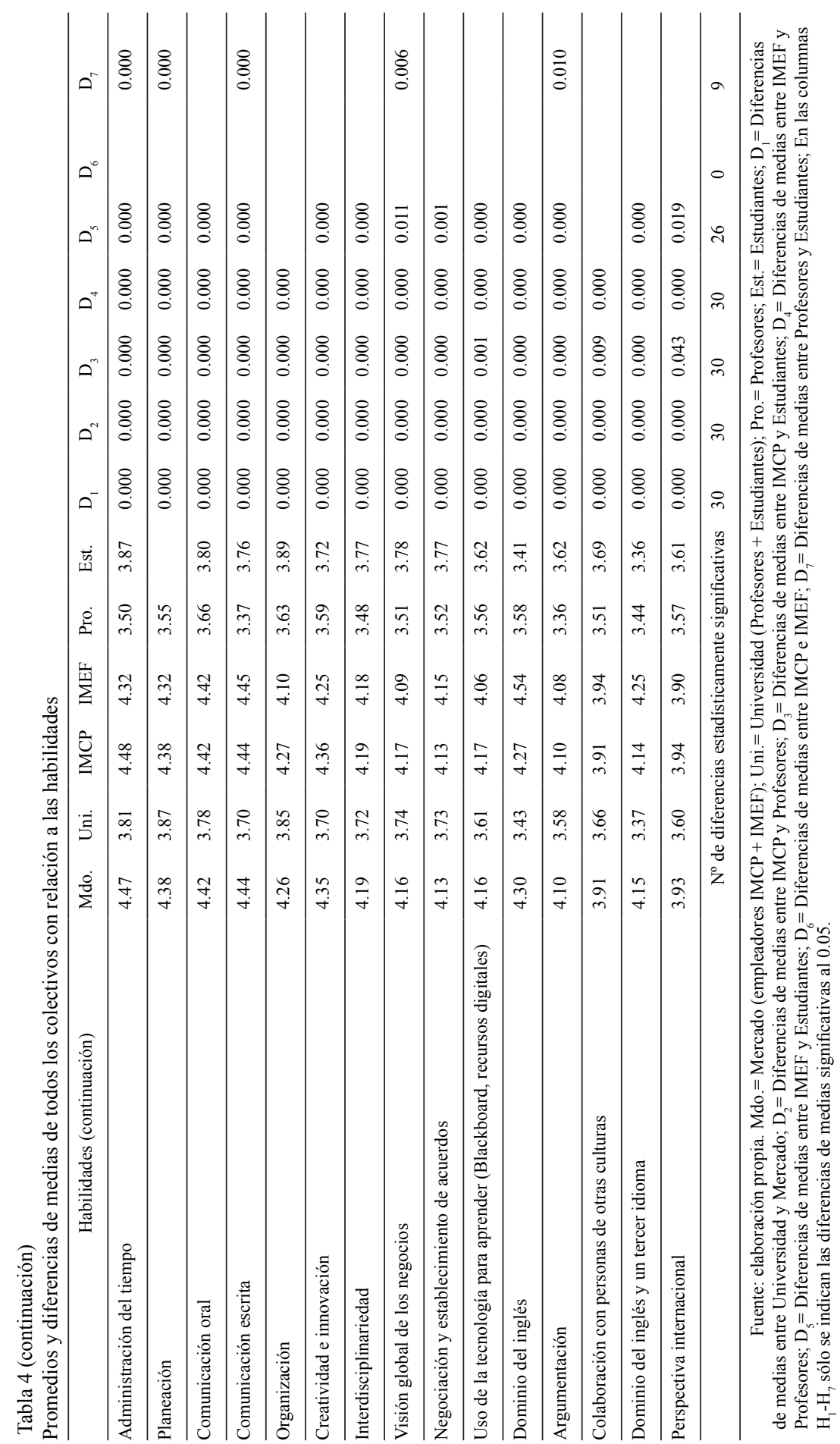




\section{Valores, ética y actitudes}

En la Tabla 5 se presentan los resultados obtenidos con relación al nivel de conciencia que un recién egresado universitario en CPF debe tener sobre un total de 7 valores, ética y actitudes para poder desempeñar adecuadamente su actividad profesional. Los datos están divididos según procedan de la opinión del mercado (empleadores del IMCP y del IMEF) o de la universidad (profesores y estudiantes), y ordenados de mayor a menor diferencia de medias entre ambos colectivos.

También en este caso se pone de manifiesto que los 7 valores, ética y actitudes considerados en esta investigación presentan diferencias significativas entre el nivel de exigencia del mercado y el nivel de capacitación de la universidad, con puntuaciones medias superiores en todos los casos a favor del mercado. Esta situación es un agregado más a las debilidades de formación en conocimientos y habilidades identificadas en CPF de las universidades en México actualmente, en particular en cuestiones como "Cumplimiento de leyes, regulaciones y normas" y "Objetividad e independencia".

Tabla 5

Promedios y diferencias de medias de todos los colectivos con relación a los valores, ética y actitudes

\begin{tabular}{|c|c|c|c|c|c|c|c|c|c|c|c|c|c|}
\hline Valores, ética y actitudes & Mdo. & Uni. & IMCP & IMEF & Pro. & Est. & $\mathrm{D}_{1}$ & $\mathrm{D}_{2}$ & $\mathrm{D}_{3}$ & $\mathrm{D}_{4}$ & $\mathrm{D}_{5}$ & $\mathrm{D}_{6}$ & $\mathrm{D}_{7}$ \\
\hline Objetividad e independencia & 4.73 & 4.08 & 4.74 & 4.67 & 3.84 & 4.12 & 0.000 & 0.000 & 0.000 & 0.000 & 0.000 & & \\
\hline $\begin{array}{l}\text { Cumplimiento de leyes, } \\
\text { regulaciones y normas }\end{array}$ & 4.76 & 4.04 & 4.76 & 4.76 & 4.05 & 4.04 & 0.000 & 0.000 & 0.000 & 0.000 & 0.000 & & \\
\hline $\begin{array}{l}\text { Respeto a las instituciones } \\
\text { y normas de convivencia } \\
\text { pública }\end{array}$ & 4.53 & 4.07 & 4.54 & 4.48 & 3.99 & 4.09 & 0.000 & 0.000 & 0.000 & 0.042 & & & \\
\hline $\begin{array}{l}\text { Tolerancia a las diferencias } \\
\text { entre las personas }\end{array}$ & 4.48 & 4.01 & 4.49 & 4.35 & 3.89 & 4.04 & 0.000 & 0.000 & 0.000 & 0.000 & 0.004 & & \\
\hline $\begin{array}{l}\text { Comportamiento } \\
\text { socialmente responsable }\end{array}$ & 4.50 & 3.99 & 4.52 & 4.35 & 3.79 & 4.02 & 0.000 & 0.000 & 0.000 & 0.000 & 0.002 & & 0.017 \\
\hline $\begin{array}{l}\text { Compromiso con el } \\
\text { desarrollo sustentable }\end{array}$ & 4.42 & 3.88 & 4.43 & 4.32 & 3.73 & 3.91 & 0.000 & 0.000 & 0.000 & 0.000 & 0.000 & & \\
\hline Defensa del interés público & 4.30 & 3.85 & 4.33 & 4.04 & 3.55 & 3.91 & 0.000 & 0.000 & 0.000 & 0.000 & & 0.017 & 0.000 \\
\hline${ }^{\circ}$ de diferencias & & & & & & & 7 & 7 & 7 & 7 & 5 & 1 & 2 \\
\hline
\end{tabular}

Fuente: elaboración propia. Mdo.= Mercado (empleadores IMCP + IMEF); Uni.= Universidad (Profesores + Estudiantes); Pro.= Profesores; Est.= Estudiantes; $\mathrm{D}_{1}=$ Diferencias de medias entre Universidad y Mercado; $\mathrm{D}_{2}=$ Diferencias de medias entre IMCP y Profesores; $\mathrm{D}_{3}=$ Diferencias de medias entre IMCP y Estudiantes; $\mathrm{D}_{4}=$ Diferencias de medias entre IMEF y Profesores; $\mathrm{D}_{5}=$ Diferencias de medias entre IMEF y Estudiantes; $\mathrm{D}_{6}=$ Diferencias de medias entre IMCP e IMEF; $\mathrm{D}_{7}=$ Diferencias de medias entre Profesores y Estudiantes; En las columnas $\mathrm{H}_{1}-\mathrm{H}_{7}$ sólo se indican las diferencias de medias significativas al 0.05 .

\section{Análisis cualitativo}

Como primera aproximación a los resultados del grupo focal se presenta la figura 1, en el que se recogen los términos a los que se alude con mayor frecuencia durante la sesión. En concreto, las palabras "conocimiento" y "valores" son las dos más referenciados, seguidos de "habilidades", "estudiante" y "actitudes". 


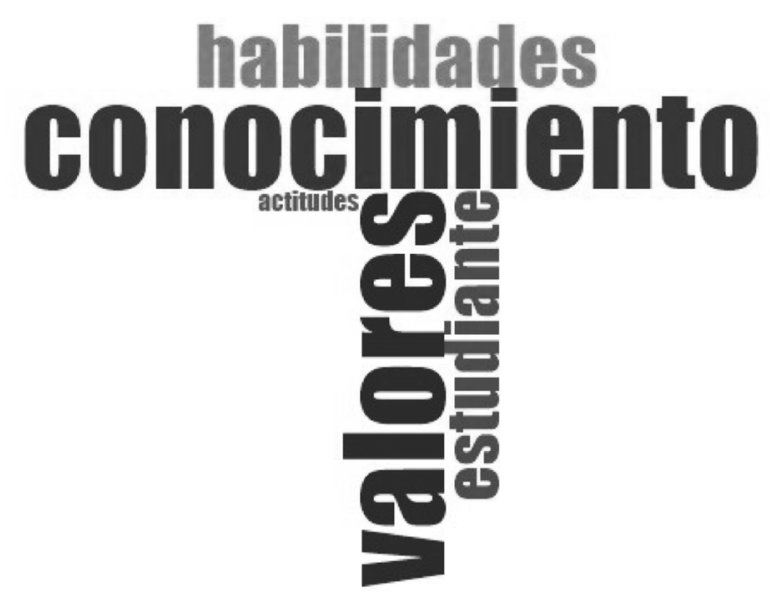

Figura. 1. Términos más mencionados por el grupo focal Fuente: elaboración propia con datos de NVivo v. 11.

Con relación a la primera cuestión planteada al grupo focal, los tres empleadores están de acuerdo con los resultados de la encuesta respecto al orden de importancia concedido a las tres vertientes de las competencias: en primer lugar los valores, ética y actitudes, seguido de las habilidades y en tercer lugar los conocimientos. Éstas son algunas de las consideraciones más interesantes hechas por ellos sobre este tema:

"Siempre he dicho que las actitudes son más importantes que las aptitudes, porque las actitudes son las que te dan la capacidad para desarrollar las habilidades. Llegar temprano a trabajar; no estar pensando en que ya son las seis de la tarde y ya me tengo que ir, sino pensar en terminar el trabajo y en hacerlo mejor; si no sé, preguntar; etc.” (Empleador 1).

"Los recién egresados llegan con la idea de que ya terminé y ya soy dueño del mundo, entonces puedo hacer lo que yo quiera. Y muchos de los problemas están en las actitudes, o sea en los valores. El hecho de que no se respeten las jerarquías y simplemente se vea a todos por igual, el lenguaje con el que se expresan también dice mucho, porque en muchas ocasiones se siguen comportando como si estuvieran todavía en las aulas educativas" (Empleador 2).

"Por supuesto que es muy importante la formación académica, pero la actitud es más importante, porque vas a aprender toda tu vida. No es nada más lo que aprendes en la Universidad lo que después vas a utilizar, sino que lo que tú aprendes ya en la práctica va a ir encima de lo que tú ya aprendiste antes, y vas a incrementar ese conocimiento. Pero para tener una posición de más responsabilidad, o para lograr cualquier objetivo que tú tengas, vas a tener que tener la suma de lo que estamos diciendo: las actitudes, las habilidades y el conocimiento. Y creo que van en ese orden que estamos diciendo" (Empleador 3).

En su turno de intervención, uno de los profesores hace el siguiente comentario de interés: 
"Bueno, en principio yo estoy de acuerdo de la importancia de las actitudes. Aunque sí considero conveniente que exista un balance, porque en nuestra rama sí hay evaluaciones de entrada con diferentes empleadores que son brutalmente técnicas” (Profesor 2).

Ahora bien, con relación a la formación en valores, ética y actitudes, y enlazando con la segunda cuestión planteada al grupo focal, existe cierta discrepancia respecto al hecho de que deba responsabilizarse a la universidad. Así, por ejemplo, se dice:

“Las actitudes y los valores son más de la familia o, mejor dicho, son más innatos de cada persona” (Empleador 3).

"No coincido al 100\% en pensar que no es responsabilidad de la Universidad formar a los estudiantes en valores/actitudes y habilidades. Efectivamente, coincido en que la familia es fundamental y la persona trae su propia personalidad, pero creo que también como profesores sí debe ser responsabilidad nuestra conjuntar esfuerzos para reforzar a través de ejemplos y comunicación con el estudiante hacerle ver que si no tiene esos valores desarrollados (ética, profesionalismo, entrega, honestidad, etc.) pues no va a llegar lejos", (Profesor 3).

"Los valores son algo que nos va a marcar mucho. Vienen de la familia, claro, pero también nosotros como universidad tenemos que fomentarlos y hacer que estén siempre ahí" (Profesor 1).

"La institución académica debería hacer un poquito más por reforzar los valores y las actitudes, porque tenemos conocimientos, aunque muchas veces un poco vagos, producto de la memorización” (Estudiante 3).

Con relación a este último punto de la memorización para la adquisición de conocimientos, parece pertinente destacar la siguiente observación:

"Yo creo que lo que se nos olvida a veces es que comprendan el conocimiento, la semillita, y una vez que tienes la semillita, con las habilidades y los valores que vamos a desarrollar entonces esa semillita se va ir al lado que se tenga que ir" (Profesor 1)

Preguntados los empleadores por lo que buscan a la hora de incorporar recién egresados a sus equipos de trabajo, estos son algunos de sus comentarios:

"Que tengan una carrera afín con una calificación aceptable, acompañada de actividades extracurriculares, y que tengan una experiencia internacional, no sólo otro idioma, sino que tengan la experiencia cultural de otro país” (Profesional 1).

"Yo buscaría una persona que venga equilibrada, que tuviera ese balance. También que fuera una persona con la capacidad para pensar, analizar e interpretar por sí misma, y así resolver situaciones y problemas” (Profesional 2). 
"Yo coincido con eso. O sea, sí buscamos gente que tenga balanceados estos tres componentes que estamos diciendo. Y creo que dependiendo de la posición o del puesto que estés contratando, pues es distinto lo que vas a requerir que esté más fuerte en esa persona" (Profesional 3).

\section{Conclusiones}

Con este trabajo se contribuye a la escasa literatura empírica previa sobre el estudio de las competencias relevantes para el desempeño profesional de la CPF en el ámbito latinoamericano, y particularmente en el caso de México (Montoya, Farías y Mancina, 2013). En la misma línea de investigaciones previas, los resultados obtenidos son indicativos de una considerable brecha entre la oferta formativa de las universidades y las demandas del mercado laboral en cuanto a conocimientos, habilidades y valores, ética y actitudes. De este modo, se aporta un mejor conocimiento de la situación en la actualidad, que deriva en importantes implicaciones prácticas, tanto a nivel académico como gerencial.

En primer lugar, este trabajo resulta de interés para los responsables de diseñar los programas académicos en las universidades, así como para los profesores encargados de impartir docencia dentro de ellas, ya que se proporciona información que les puede servir de guía para revisar las estrategias y acciones de formación a las necesidades actuales de la práctica laboral. Por otra parte, desde el plano empresarial este trabajo es relevante para organismos como la IFAC, en el ámbito internacional, y el IMCP y el IMEF, en el ámbito nacional de México, de cara a reexaminar y adecuar los distintos marcos normativos que regulan la formación para el ejercicio y el desempeño profesional de la CPF.

A este respecto, es fundamental lograr un mayor acercamiento y diálogo en el binomio universidad-empresa, a fin de evitar asimetrías de información entre los dos ámbitos y desajustes relacionados con la empleabilidad de los egresados como los mostrados en este estudio (Frecka y Reckers 2010; Hancock, Howieson, Kavanagh, Kent, Tempone y Segal, 2010). Además, es necesario fomentar la adquisición de experiencia práctica durante los estudios universitarios (Paisey y Paisey, 2010), así como una mayor implantación y utilización de metodologías de enseñanza activa, como el Aprendizaje Orientado a Proyectos, el Aprendizaje Basado en Problemas o el Estudio de Casos, las cuales favorecen el desarrollo de competencias por medio de la concesión de un mayor protagonismo y de la exigencia de más trabajo autónomo a los estudiantes (Stoner y Milner, 2010; Robledo, Fidalgo, Arias y Álvarez, 2015). Todo ello posibilitaría a las universidades contar con egresados mejor preparados, y a las empresas y despachos profesionales recibir en sus procesos de reclutamiento a personas con un perfil más alineado con sus necesidades laborales (Apostolou, Dorminey, Hassell y Rebele, 2015).

La interpretación de todo lo anterior debe hacer con las debidas cautelas. Los resultados de la encuesta están basados en las opiniones de una muestra de miembros del IMCP y del IMEF, en la parte profesional, y de una serie de profesores y estudiantes de algunas universidades mexicanas, en la parte académica. En consecuencia, estos resultados podrían no ser extrapolables a otros países, otros colectivos similares u otro tipo de actividades profesionales en las que pudiera encajar una persona con estudios universitarios relacionados con la CPF. Futuros trabajos que se desarrollen en esta línea podrían extenderse a otros países latinoamericanos, incluso tomar en cuenta a otros colectivos, como las autoridades responsables de la regulación en el ámbito tanto académico como profesional. Además, se debe seguir profundizando en la investigación 
sobre las metodologías activas de enseñanza-aprendizaje más apropiadas para la formación de competencias relevantes en estas áreas, a fin de que los egresados de las universidades estén mejor preparados para su inserción laboral.

\section{Referencias}

Apostolou, B., Dorminey, J. W., Hassell, J. M. \& Rebele, J. E. (2015), “Accounting education literature review (20132014)", Journal of Accounting Education, 33 (2), 69-127. http://dx.doi.org/10.1016/j.jaccedu.2015.04.001

Arquero, J. L. (2000), "Capacidades no técnicas en el perfil profesional en Contabilidad: las opiniones de docentes y profesionales", Revista Española de Financiación y Contabilidad, 29 (103), 149-172.

Arquero, J. L., Donoso, J. A., Jiménez, S. A. \& González, J. M. (2009), “Análisis exploratorio del perfil demandado para Administración y Dirección de Empresas: implicaciones para el área contable”, Revista de Contabilidad, 12 (1) 43-66. doi:10.1016/S1138-4891(09)70006-8

Bartual, M. T. \& Turmo Garuz, J. (2016), "Educación superior y competencias para el empleo. El punto de vista de los empresarios", Revista Complutense de Educación, 27 (3),1211-1228. doi:10.5209/rev_RCED.2016.v27.n3.47645

Bennett, N., Dunne, E. \& Carré, C. (1999), "Patterns of core and generic skill provision in higher education", Higher Education, 37 (1), 71-93. doi:10.1023/A:1003451727126

Biemans, H., Nieuwenbuis, L., Poell, R., Mulder, M. \& Wesselink, R. (2004), "Competence based VET in the Netherland. Background and piftalls", Journal of Vocational Education and Training, 56 (2),523-538. http://dx.doi. org $/ 10.1080 / 13636820400200268$

Caballero, G., López, M. J. \& Lampón, J. (2014), “La universidad y su implicación con la empleabilidad de sus graduados", Revista Española de Investigaciones Sociológicas, 146 (1), 23-46. http://dx.doi.org/10.5477/cis/reis.146.23

Cabeza, L., Castrillón, J. \& Lombana, J. (2012), "Determinación y análisis de las competencias por fortalecer para la gestión en Colombia", Innovar, 22 (46), 5-20.

Castrillón, J., Cabeza, L. \& Lombana, J. (2015), “Competencias más importantes para la disciplina administrativa en Colombia”, Contaduría y Administración, 60 (4), 776-795. http://dx.doi.org/10.1016/j.cya.2015.07.009

Delors, J. [Coord.] (1996), La educación encierra un tesoro, Ediciones UNESCO, Madrid.

Frecka, T. J. \& Reckers, P. M. J. (2010), "Rekindling the debate: What's right and what's wrong with masters of accountancy programs: The staff auditor's perspective", Issues in Accounting Education, 25 (2), 215-226. http:// dx.doi.org/10.2308/iace.2010.25.2.215

Hancock, P., Howieson, B., Kavanagh, M., Kent, J., Tempone, I. \& Segal, N. (2010), Accounting for the future, Accounting Education at a Crossroad in 2010 (pp. 54-62). Australia: The Institute of Chartered Accountants in Australia.

International Federation of Accountants. (2014), Handbook of International Education Pronouncements. New York: International Accounting Education Standards Board, June.

Ivankovich, C. I. \& Araya, Y. (2011), "Focus groups: Técnica de investigación cualitativa en investigación de mercados", Ciencias Económicas, 29 (1), 545-554.

Kavanagh, M. H., \& Drennan, L. (2008), "What skills and attributes does an accounting graduate need? Evidence from student perceptions and employer expectations", Accounting and Finance, 48 (2), 279-300. http://dx.doi. org/10.1111/j.1467-629X.2007.00245.x

Lin, P., Krishnan, S. \& Grace, D. (2013), "The effect of experience on perceived communication skills: Comparisons between accounting professionals and students", Advances in Accounting Education: Teaching and Curriculum Innovations, 14, 131-152. doi: 10.1108/S1085-4622(2013)0000014012

Martínez, P. \& Echeverría, B. (2009), "Formación basada en competencias", Revista de Investigación Educativa, 27 (1), 125-147. doi: 10.6018/rie.27.1.94331

Marzo, M., Pedraja, M. \& Rivera, P. (2009), “Curricular profile of university graduates versus business demands: Is there a fit or mismatch in Spain?”, Education and Training, 51 (1), 56-69. http://dx.doi.org/10.1108/00400910910931832

Montoya del Corte, J., Farías Martínez, Mª G. \& Mancilla Rendón, M ${ }^{a}$ E. (2013), “Competencias profesionales de los egresados en Contaduría Pública”, Contaduría Pública - Instituto Mexicano de Contadores Públicos, No. 493 , pp. 30-33. 
McLagan, P. (1997), “Competencies: The Next Generation”, Training and Development, 51 (5), 40-47.

Nunnally, J. C. (1978), Psychometric Theory. New York: McGraw Hill.

Paisey, C. y Paisey, N. J. (2010), "Developing skills via work placements in accounting: Student and employer views", Accounting Forum, 34 (2), 89-108. http://dx.doi.org/10.1016/j.accfor.2009.06.001

Pan, P. \& Perera, H. (2012), "Market relevance of university accounting programs: Evidence from Australia", Accounting Forum, 36 (2), 91-108. http://dx.doi.org/10.1016/j.accfor.2011.11.001

Pujol-Jover, M., Riera-Prunera, C. \& Abio, G. (2015), “Competences acquisition of university students: Do they match job market's needs?", Intangible Capital, 11 (4), 612-626. http://dx.doi.org/10.3926/ic.625

Robledo, P., Fidalgo, R., Arias, O. \& Álvarez, M. (2015), "Percepción de los estudiantes sobre el desarrollo de competencias a través de diferentes metodologías activas", Revista de Investigación Educativa, 33 (2), 369-383. http:// dx.doi.org/10.6018/rie.33.2.201381

Rubio, R. M. (2005), "Aims and functions of the university in the European Space of Higher Education", Revista Española de Pedagogía, 63 (230), 17-42.

Segars, A. H. (1997), “Assessing the unidimensionality of measurement: A paradigm and illustration within the context of information Systems research", Omega, 25 (1), 107-121. doi: 10.1016/S0305-0483(96)00051-5

Senik, R., Broad, M., Mat, N. \& Kadir, S. A. (2013), "Information technology (IT) knowledge and skills of accounting graduates: Does an expectation gap exist?”, Journal Pengurusan, (38), 87-100.

Stoner, G. \& Milner, M. (2010), "Embedding generic employability skills in an accounting degree: Development and impediments", Accounting Education, 19 (1-2), 123-138. http://dx.doi.org/10.1080/09639280902888229

Tempone, I., Kavanagh, M., Segal, N., Hancock, P., Howieson, B. \& Kent, J. (2012), "Desirable generic attributes for accounting graduates into the twenty-first century: The views of employers", Accounting Research Journal, 25 (1), 41-55. http://dx.doi.org/10.1108/10309611211244519

Varela, O. (2003), “Competencias y desempeño humano: mito o realidad”, Academia Revista Latinoamericana de Administración, (30), 97-108. 